Document downloaded from:

http://hdl.handle.net/10251/45104

This paper must be cited as:

Garrido Tejero, A.; Morales, L.; Fernandez, S.; Onaindia De La Rivaherrera, E.; Morales, L.; Borrajo, D.; Castillo, L. (2013). On the automatic compilation of e-learning models to planning. Knowledge Engineering Review. 28(2):121-136. doi:10.1017/S0269888912000380.

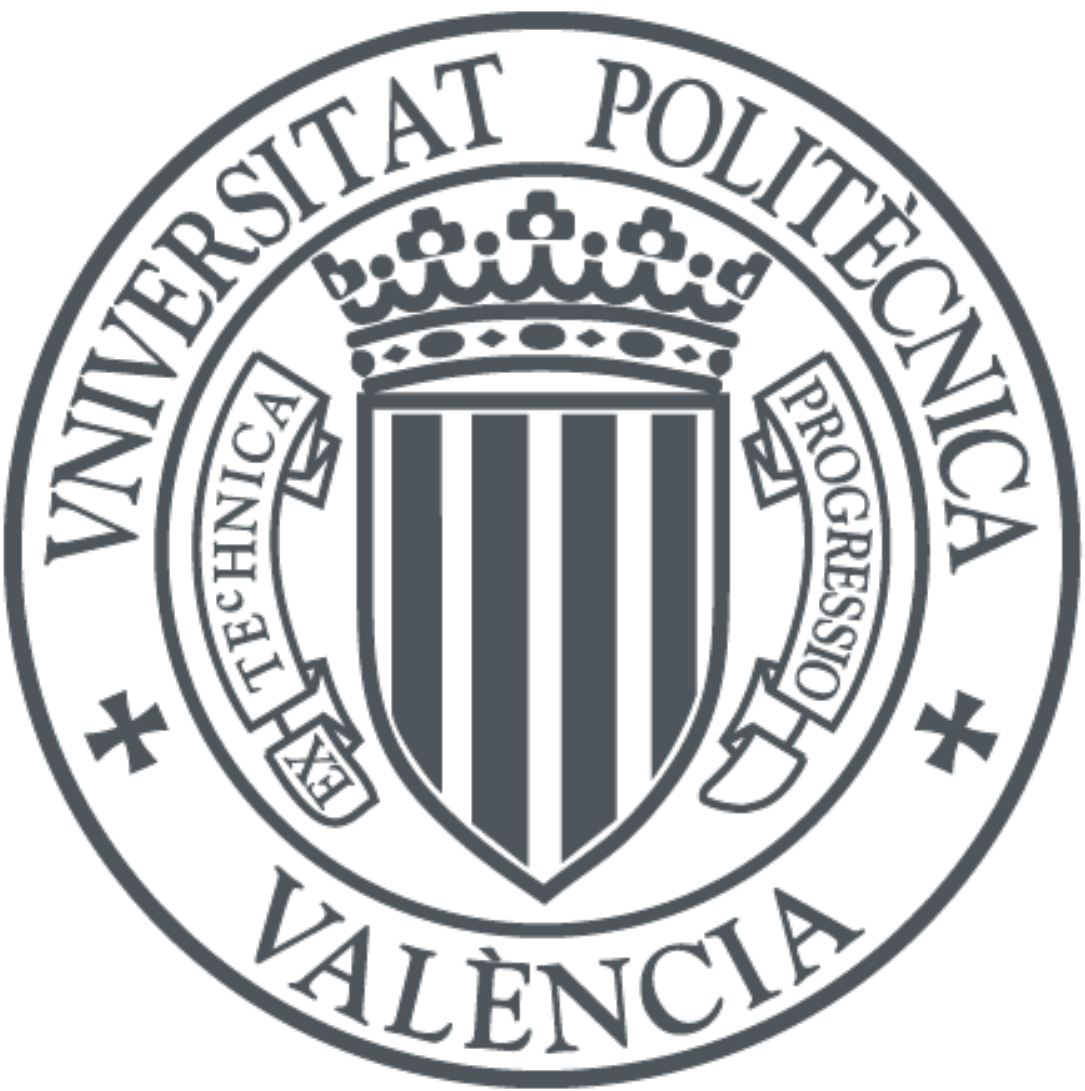

The final publication is available at

http://dx.doi.org/10.1017/S0269888912000380

Copyright Cambridge University Press (CUP)

Additional Information 


\title{
On the automatic compilation of e-learning models to planning
}

\author{
Antonio Garrido ${ }^{1}$, Susana Fernández ${ }^{2}$, Lluvia Morales $^{3}$, Eva Onaindía ${ }^{1}$, Daniel \\ Borrajo $^{2}$ and Luis Castillo ${ }^{3}$ \\ ${ }^{1}$ Universidad Politecnica de Valencia, Camino de Vera $s / n, 46071$ (Valencia). Spain \\ E-mail: \{agarridot, onaindia\}@dsic.upv.es \\ ${ }^{2}$ Universidad Carlos III de Madrid, Avda. de la Universidad 30, 28911 (Leganés, Madrid). Spain \\ E-mail: \{sfarregu,dborrajo\}@inf.uc3m.es \\ ${ }^{3}$ Universidad de Granada, C/ Periodista Daniel Saucedo Aranda s/n, 18071 (Granada). Spain \\ E-mail: \{lluviamorales, l.castillo\}@decsai.ugr.es
}

\begin{abstract}
This paper presents a general approach to automatically compile e-learning models to planning, allowing us to easily generate plans, in the form of learning designs, by using existing domainindependent planners. The idea is to compile, first, a course defined in a standard e-learning language into a planning domain, and, second, a file containing students learning information into a planning problem. We provide a common compilation and extend it to three particular approaches that cover a full spectrum of planning paradigms, which increases the possibilities of using current planners: i) hierarchical, ii) including PDDL actions with conditional effects, and iii) including PDDL durative actions. The learning designs are automatically generated from the plans and can be uploaded, and subsequently executed, by learning management platforms. We also provide an extensive analysis of the e-learning metadata specification required for planning, and the pros and cons on the knowledge engineering procedures used in each of the three compilations. Finally, we include some qualitative and quantitative experimentation of the compilations in several domain-independent planners to measure its scalability and applicability.
\end{abstract}

\section{Introduction}

E-learning is becoming a high-impact innovative topic as it offers a promising way to facilitate and enhance the learning process by combining learning objects (LOs), typically any sort of digital resource, to create flexible courses. This flexibility and the availability of automatic tools to create learning routes allows e-learning experts to focus on content authoring, and relieves them of the burden of manually composing standard documents, such as learning designs, that fit the needs of each student in a course.

Creating tailored routes of LOs, according to student profile preferences and/or pedagogical theories, is a subject which has been studied in depth in the Planning \& Scheduling (P\&S) community within the last years (Castillo, et al. 2010, Mohan, et al. 2003, Kontopoulos, et al. 2008, Ullrich \& Melis 2009). Generating these routes depends on many elements: LOs prerequisites/outcomes, LOs duration, available resources, and even collaboration and interaction between tutors and students, which make the problem very interesting from the P\&S perspective. However, acquiring information from educational domains to represent it as a planning domain is not a straightforward task because pedagogical theories are usually hard to model in practice. The main reasons for this are: i) there exist essential elements (e.g. soft and hard requirements among LOs, deadlines, strong interaction between LOs or students, etc.) for the success of the learning process, but are not thoroughly modelled in traditional e-learning settings; ii) people give different meanings and uses to the standards specifications, mainly in terms of the relations 
among LOs, given that standards provide some flexibility on how to represent knowledge; and iii) there is a great variety of planning paradigms which support different aspects of expressiveness. In this paper we explicitly address these drawbacks by providing an exhaustive mapping to interpret LO-metadata in terms of AI planning, which can be easily extended to support more complex features, not usually modelled in e-learning, and can be applied in different planning paradigms.

Leaving the complex pedagogical decisions aside, in this paper we propose an alternative to previous work that focuses on solving these three difficulties. We extend the work in (Garrido, et al. 2009), presenting a knowledge engineering approach to compile information about course content and learners, subject to e-learning standards (IMSMD 2003, IMSLD 2003, SCORM 2004), and translating this information into a planning domain and problem. The planning instances are used to automatically generate plans, customised routes of LOs, that are translated into an IMS-LD standard document (IMSLD 2003). In essence, this paper contributes with:

- An automated translation of e-learning templates into a general compilation, further extended with three different PDDL (Planning Domain Definition Language (Fox \& Long 2003)) versions: i) hierarchical, ii) PDDL-conditional, and iii) PDDL-temporal. This allows us to use our compilations in virtually any current planner.

- An effective use of planning technology to generate learning designs that best suit students learning goals. Planners focus on finding the adequate LOs combination for students adaptation, thus promoting a more personalised access to the LOs.

- A translator that parses the resulting plans and generates the IMS-LD standard document to be uploaded to Learning Management Systems (LMSs), thus closing the e-learning cycle.

This paper is structured as follows. Section 2 introduces some related work on courseware generation using planning techniques, some basic description on e-learning standards and motivates our work. Section 3 analyses how to model learning designs in planning, and presents the compilation of e-learning standards into planning domains and problems. Experimental results, by using different planners, are detailed in section 4 . Finally, section 5 concludes the paper and addresses some future work.

\section{Combining E-learning and AI planning. Motivation}

There are many approaches in the adaptive hypermedia community that combine instructional knowledge and planning techniques to deal with the automation of courseware generation. Some of them introduce HTN planners to represent pedagogical objectives to find a tailored course structure, such as (Méndez, et al. 2005) and (Sicilia, et al. 2006), which provides a theoretical proposal. To our knowledge, the former does not integrate its results in an e-learning platform, whereas the latter only provides LOM translations for HTN planning. The approach in (Ullrich \& Melis 2009) is complete and has been put into practice specially for retrieving LOs from external repositories. Basically, it takes a LOM subset to be integrated into a particular ontology with additional LO information by means of a specific intelligent tutoring system, thus making it incompatible with other learning systems. According to (Ullrich \& Melis 2009), they can map the final plan into IMS-CP or SCORM but not the IMS-LD model itself and they do not use standards in the definition of the student profile. Others use state-based planners that implement metrics to measure the adaptation to a specific learning style (Boticario \& Santos 2007, Limongelli, et al. 2008), but without taking into consideration other profile features and different learning styles theories. Finally, others incorporate machine learning techniques to assist content providers when constructing LOs that comply with an ontology, concerning objectives and prerequisites, as can be seen in (Camacho, et al. 2007, Kontopoulos et al. 2008) with CAMOU and PASER, respectively, but again, they do not use e-learning standards and usually focus on a particular planner. Despite the relative success of these approaches, they are usually limited to a specifically designed ontology and planning paradigm. Our main motivation (and contribution) is to overcome such limitations. 


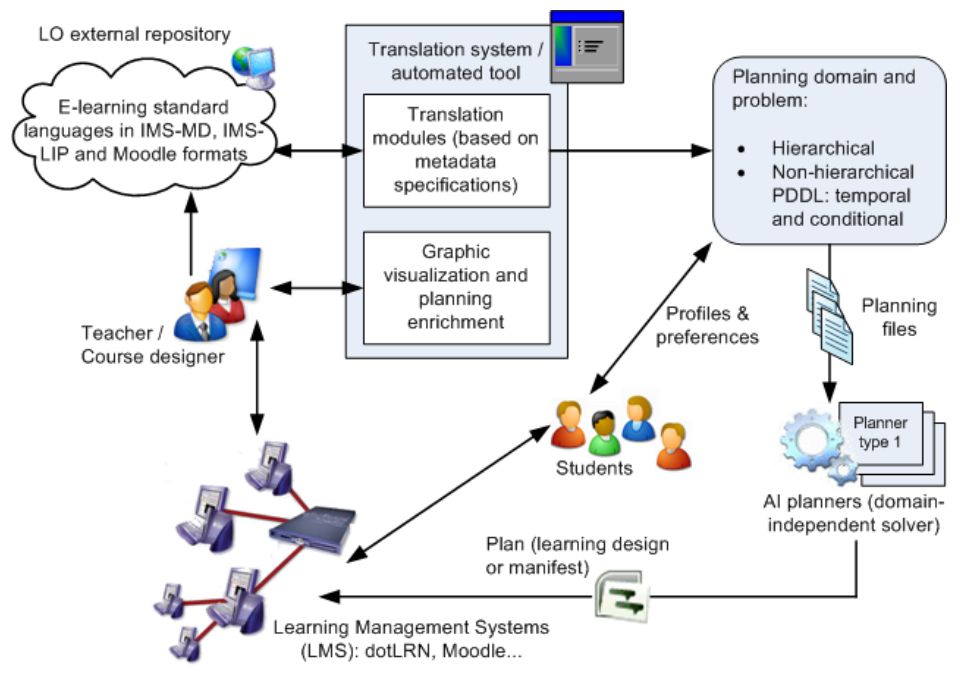

Figure 1 Overview of our entire system.

First, our approach is entirely based on well-known e-learning standards, and not on particular or ad-hoc ontologies. We use the IMS standard specification (http://www.imsglobal.org) supported by major LMSs such as Moodle (http://moodle.org) and $\operatorname{dotLRN}$ (http://dotlrn.org), which includes:

- IMS-MD to describe LOs and their relations (see example in Figure 2D;

- IMS-LIP to model the student profile (see example in Table 2 below); and

- IMS-LD to sequence the LOs according to the students profiles (see section 3.4).

Second, we use a pedagogical theory based upon learning styles, but other adaptation criteria (see (Essalmi, et al. 2010)) can be easily modelled as well. Hence, our approach provides the basis for education experts to easily experiment with different learning theories. The overall idea is to make the contents authoring easier and more adequate. As shown in Figure 1 , the teachers design, from scratch or by reusing LO collections, an e-learning course. The course designer may use our authoring tool (see (Garrido et al. 2009)) to enrich the LO metadata by visually adding/deleting relations, or even associating resources and costs to the LOs. This is not thoroughly modelled in traditional e-learning settings, but it makes the use of planning techniques more flexible; obviously, the enrichment of the metadata in our tool is entirely optional. The information about the course is profile-independent, so it is valid for students with different learning styles. Once the personal characteristics, background and preferences of the students are modelled, our translation system automatically generates the planning domain and problem files according to three different planning paradigms.

Third, our approach is appropriate for most of the existing planners, acting as domainindependent solvers. This is a clear advantage as we are not restricted to one particular solver. Once the solver generates a plan, as a LO route per student, it is subsequently compiled as an IMS standard learning design (IMS-LD). Finally, the result is uploaded to a LMS, which manages the administration, display, tracking and navigation of the contents of the learning design, thus closing the cycle with standards.

\section{Compiling E-Learning Models for Planning}

In our compilation we consider the repository of LOs as the planning domain, whereas the relevant students characteristics and interests represent the planning problem. On the other hand, the learning design represents the solution plan. In all cases, domain, problem and plan, 
the information required for the compilation is fully automated by extracting the metadata specifications from the e-learning standards.

\subsection{Metadata for Planning Domains}

A learning design is defined by a set of LOs, which are usually encoded as XML schemata (see Figure 22. For example, in a course for learning Discrete-Maths, there may be a task for learning Boolean-Algebra. And there could be several LOs to accomplish it, such as playing a visual presentation, reading the introduction text from a textbook, or solving an exercise. Although it may be enough for the student to use only one of these objects to accomplish the task, it is still possible to use more than one, thus improving the overall utility (reward) of the learning process. And this usually depends on how the LOs are described by their metadata set:

- LOs are more or less appropriate to students depending on their learning styles. We use two well-known theories to determine and classify students, the Felder learning style (Felder 1996), and the Honey-Alonso on $\mathrm{g}^{2}$ (Alonso \& Honey 2002), but any other can be used to describe new ordering rules or utilities. Thus, we use the representation of the pedagogical knowledge as defined in the metadata.

- LOs have dependency relations among them. For instance, before learning about Discrete-Maths, the student should have some knowledge on Boolean-Algebra. We support four types of relations that include hierarchical structures and content ordering relations. The hierarchical structures use the IsPartOf relation, which represents a hierarchical aggregation of LOs. Additionally, there are three types of causal dependencies, Requires, IsBasedOn and References. We interpret the first two relations as hard preconditions. In the case of the Required elements, all of them (conjunctively) have to be completed before initiating a new LO: if 'A Requires B' and 'A Requires C', both B and C need to be finished before doing A. In the case of the IsBasedOn elements, at least one of them (disjunctively) has to be completed: if 'A IsBasedOn B' and 'A IsBasedOn C', only B or C must be completed before initiating A. On the other hand, the course designer might also recommend (soft requirement) other previous LOs by using the References relation.

- Each LO takes a standard time (duration) to fulfill, namely typical learning time. However, only primitive LOs have duration, as the duration of aggregations is derived from their set of aggregated LOs (e.g. Discrete-Maths in Figure 2).

- Each LO belongs to a learning resource type, such as a lecture, narrative text, diagram, etc. This does not seem to be very relevant for planning, but according to education experts the learning resource type highly interacts, positive or negatively, with the student profile. For instance, a lecture is very recommendable for Felder verbal students but not for visual ones; and just the opposite holds for a diagram. Or LOs can be displayed in different orders; e.g. for a Honey-Alonso pragmatic student, an experiment must be displayed before a narrative text, but for a theoretical student this order must be inverted. The main inconvenient here is that these resource types combine constructivist and traditional didactic approaches (Ullrich 2008). That is, some describe the format of a resource, but others cover the instructional type, which represents different dimensions - there is a mix of pedagogical and technical/presentation information. Therefore, the learning resource type may fail in representing a sufficiently precise compilation if, for instance, a lecture contains an image or diagram, or an experiment is described as a text. This may become problematic, but we rely our technical compilation on the resource type, and delegate its significance entirely to the LO designer, who is the real expert in pedagogic matters.

\footnotetext{
${ }^{1}$ Felder learning styles comprise four dimensions: perception (sensitive/intuitive), processing (active/reflective), input (visual/verbal) and understanding (sequential/global).

${ }^{2}$ This learning styles theory considers four styles that can be parallelized; for instance pragmatic/active and reflexive/theoretical can be taken as two styles.
} 


\begin{tabular}{|c|c|}
\hline tem & PDDL action entry \\
\hline general $/\{$ identifier or title $\}$ & $\begin{array}{l}\text { action-name, which may be a real, fictitious, } \\
\text { primitive or an aggregation action (also known } \\
\text { as task in HTN) }\end{array}$ \\
\hline- & $\begin{array}{l}\text { :parameters (?s - student), to model the } \\
\text { student }\end{array}$ \\
\hline $\begin{array}{l}\text { if the LO is a primitive LO then } \\
\text { educational/typicallearningtime } \\
\text { else }\{\text { aggregation of } L O s\} \\
\text { sum of the educational/typicallearningtime } \\
\text { given by its IsPartOf LOs }\end{array}$ & $\begin{array}{l}\text { :duration for temporal planners, or } \\
\text { :effect (increase (total_time ?s) value) } \\
\text { managed as an artificial fluent effect for } \\
\text { temporal and non-temporal metric planners }\end{array}$ \\
\hline $\begin{array}{l}\text { dependency relations: } \\
\text { type of relation: } \\
\text { switch (relation/kind/value) } \\
\text { case (Requires): conjunctive (and) precondition } \\
\text { case (IsBasedOn): disjunctive (or) precondition } \\
\text { the related LO: relation/resource/catalogentry }\end{array}$ & $\begin{array}{l}\text { :precondition } \\
\text { (and (not (action-name_done ?s)), and } \\
\text { if conjunctive precondition: (and ... } \\
\text { else-if disjunctive precondition: (or ... } \\
\text { for each catalogentry value: } \\
\quad \text { (action-value_done ?s), which creates a } \\
\text { particular ordering (also required in HTN) } \\
\text {... other optional preconditions for profile } \\
\text { adaptation taken from educational item) }\end{array}$ \\
\hline $\begin{array}{l}\text { increase a reward (utility expression) or creating a } \\
\text { particular ordering (useful in HTN) due to: } \\
\text { profile adaptation: } \\
\text { educational/learningresourcetype/value } \\
\text { additional adaptation by using a metric reward: } \\
\text { if relation/kind/value is References }\end{array}$ & $\begin{array}{l}\text { :effect } \\
\text { (and (action-name_done ?s) ..., to model } \\
\text { that the action has been done, and } \\
\text { (increase (reward ?s) value_LRT) } \\
\text { (increase (reward ?s) value_References) } \\
\text {... other optional rewards and/or costs) }\end{array}$ \\
\hline
\end{tabular}

Table 1 General LO metadata mapping to PDDL actions. Irrelevant information has been ignored.

a given LO is for a learning style. Second, the References dependency relation also may increase the reward by a given value_LRT when the student satisfies that recommendation. Again, this value can be particularised to each learning style. Optionally, the compilation can include numeric expressions or resource costs, as are common in P\&S, and even a particular sequencing used to assist hierarchical decomposition.

This mapping is very general in terms of the planning concepts. Although it comprises many features, such as durations, conjunctive and disjunctive preconditions, propositional and numericvalued representations, etc., it does not define other such as hierarchical structures. On the other side, there are currently several planning paradigms, and planners. Thus, we instantiate this general algorithm to provide specialised compilations for different planning paradigms. More particularly, we started with an extension of our general compilation to a hierarchical compilation, and then we added two approaches for PDDL compilations. The first one based on conditional effects, named conditional compilation, that encodes in one operator all the profile adaptation options. Again, as many planners do not support conditional effects, we provide an additional compilation where all operators are grounded, hierarchies are simulated by means of dummy actions and actions can be durative (temporal compilation). It is important to note that we are not proposing three different planning systems, but three compilations aiming at covering practically the full spectrum of planning paradigms that exist today.

\subsubsection{Hierarchical Domain Compilation}

This compilation extends the general compilation with the following features:

- It is based on an extended version of PDDL for HTN planning (Castillo, et al. 2006), which includes a hierarchical representation of tasks and methods within a related temporal framework that allows us to specify goal deadlines, temporal landmarking between actions, and a constraint propagation engine for exploiting the causal structure of plans. 


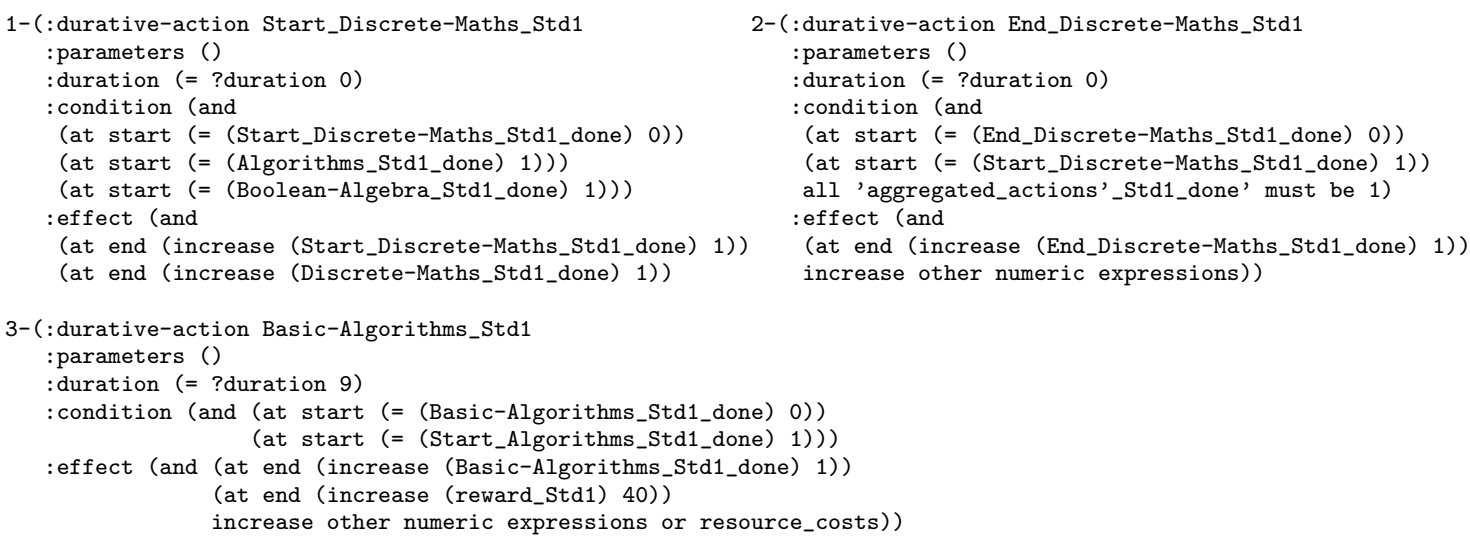

Figure 5 Durative actions for student Std1 generated for the two LOs of Figure 2 according to the PDDL-Temporal compilation. We assume in our problem that Std1 is verbal. Since the learning resource type of Basic-Algorithms is 'Narrative Text' (see Figure 2) and the student is verbal this means a reward in the learning process of 40 . The values of all the rewards are given by the educational experts.

\begin{tabular}{|c|c|}
\hline IMS-LIP & Planning problem \\
\hline identification/name/contentype/referential/indexid & :objects student-name, checking its uniqueness \\
\hline accessibility/preference/typename/typevalue/ & :inits (profile-type student-name value) \\
\hline Learner_Style_Processing/prefcode/profile-type.value & e.g. :inits (reflective student1 strong) \\
\hline $\begin{array}{l}\text { goal/typename/typevalue/course-name/contentype/temporal/ } \\
\text { typename/time_threshold/temporalfield/value }\end{array}$ & $\begin{array}{l}\text { :inits (= (time_threshold student-name) value) } \\
\text { :goals (= (course-name_done student-name) }) \\
\text { e.g. : inits (= (time_threshold student } 1) \text { 3800) } \\
\text { e.g. :goals (course-name_done student1) }\end{array}$ \\
\hline $\begin{array}{l}\text { activity/typename/typevalue/task/learningactivityref/ } \\
\text { text/object-name }\end{array}$ & $\begin{array}{l}\text { :objects object-name } \\
\text { :inits (known student-name object-name) } \\
\text { e.g. : objects graph_theory } \\
\text { e.g. :inits (known student1 graph_theory) }\end{array}$ \\
\hline accessibility/language/typename/type & $\begin{array}{l}\text { :inits (language_level lang-value } \\
\text { student-name high) } \\
\text { e.g. : inits (language_level English student1 } \\
\text { high) }\end{array}$ \\
\hline $\begin{array}{l}\text { competency/contentype/referential/indexid/performance-level/ } \\
\text { description/short/value }\end{array}$ & $\begin{array}{l}\text { :inits (performance_level student-name high) } \\
\text { e.g. :inits (performance_level student1 high) }\end{array}$ \\
\hline
\end{tabular}

Table 2 IMS-LIP mapping to a problem compilation. Irrelevant information has been ignored.

is valid for our three approaches, although the translation differs slightly for the temporal compilation, where the problem is (interactively) generated together with the domain because of the grounded approach (Garrido et al. 2009).

The planning problem includes propositions to represent the objects, the initial state, the goals and the metric to optimise. The objects represent the students information for the learning design and their previous knowledge. The initial state represents the students profile, the initial values of the fluents, the language of the course, and some other information (e.g. student performance, special equipment, availability, etc.). The goal is to pass the entire course or a part of it. Table 2 shows the mapping to automatically translate an IMS-LIP structure into a planning problem. The first column represents the IMS-LIP entry and the second one the corresponding item in the planning problem. As can be seen, the problem compilation is simpler than the domain compilation, as it basically consists in generating the initial values and goals for the objects.

\subsection{Plans compilation}

After compiling the planning domain and problem, we run a domain-independent planner to find a plan. The quality of the plan itself depends on the planner quality; some planners are optimal and return the best solution, but others return just one solution. Consequently, the domain/problem compilation has not a relevant impact in that quality. In this compilation, we translate the plan into the e-learning standard IMS-LD. Each plan represents the learning design 
as a route of LOs that best suits the student, which will be later displayed in a LMS. Since current LMSs support different languages, we have implemented translators for two of the most common systems: IMS-LD for dotLRN, and Moodle templates. In the first case, we create a zip file that contains the input resources (LOs) as well as the learning design as an XML file. Our algorithm compiles the next six items in the XML file:

- Goals, which are taken from the goals of the planning problem, usually the effects of the final action of any domain.

- Prerequisites, which are taken from the initial conditions on previous knowledge that is required to follow this course. They are the links to LOs or objectives of other courses, which allow us to perform multiple course planning in the future.

- Roles. In this case, the only role is the student for whom the learning design is generated.

- Activities, which are taken from the plan. Iterating for each action, an IMS-LD activity entry is generated, which also includes a link to the corresponding LO. Obviously, all fictitious actions used during planning are now omitted.

- Activity structure, which relates to the plan itself and its route of actions. Given that the IMS-LD standard allows other control structures, such as conditional plans with branches, in the future we will study how to generate conditional plans and the impact it has on the fact that students may execute different alternatives.

- Resources. For each LO in the input IMS-MD, a resource, that can or not be used in the plan, is defined.

The second translator compiles the plan into a Moodle template that describes an XML file which is related to the course previously implemented in Moodle. The compilation algorithm is straightforward, as the XML document simply contains the student identifier and an enumeration of the LOs to be executed. The sequence in which LOs appear in the document corresponds to the particular ordering for each student, which helps the student explore and navigate through the course.

\subsection{Approaches Comparison}

Table 3 shows the differences between the three compilations from a knowledge engineering perspective based on several characteristics. Although the underlying semantics in the three compilations is the same, each compilation adapts more naturally to each learning language. More specifically, the Hierarchical compilation adapts better to the Moodle format in terms of domain and problem, whereas the Conditional one adapts better to the IMS-MD standard. This is because of the nature of the Moodle courses, which usually focus more on the inner structure of the course, rather than IMS-MD, which focuses more on the definition of particular LOs and leaves apart somewhat the relations between LOs. On the other hand, the Temporal compilation accepts both formats, as it manages both hierarchical and flat structures. The planning goals, deadlines (time limit devoted to the course) and prerequisites (student previous knowledge for the course) are defined in the IMS-LIP for the Hierarchical and Conditional approaches, whereas the Temporal compilation uses the data defined in the problem. The row Dependency relations considers the types of relations supported by the different approaches. Students profiles refer to the styles that are currently supported but this can be easily extended. Soft preconditions refer to the fact that the learning design can contain LOs that, without being mandatory, provide some benefit to the student. This is possible through the methods in the Hierarchical approach and through the precondition ( $<$ (total_time ?s) (time_threshold ?s)), which allows the Conditional representation to include more LOs than strictly necessary. Time management represents how the approaches deal with time. The row Metrics means whether the approach can manage quality metrics or not. The row Planner represents the planner required to solve the problems modelled by each compilation. The last row shows how the plans, which the corresponding planner generates, comply with Moodle and IMS-LD. 


\begin{tabular}{|l|l|l|l|} 
Characteristic & Hierarchical & PDDL-Conditional & PDDL-Temporal \\
\hline Domain definition & Moodle & IMS-MD & Both \\
\hline Problem definition & Moodle and IMS-LIP & IMS-LIP & Interactively \\
\hline Goal definition & IMS-LIP & LO in MD and IMS-LIP & Problem \\
\hline Deadline definition & IMS-LIP & IMS-LIP & Problem \\
\hline Prerequisite definition & IMS-LIP & IMS-LIP & Problem \\
\hline Dependency relations & All & $\begin{array}{l}\text { IsBasedOn } \\
\text { Requires }\end{array}$ & All \\
\hline Students profile & Honey-Alonso & Felder & Both \\
\hline Soft preconditions & Tasks and methods & Domain & Domain (using References) \\
\hline Time management & Durative actions & Fluent & Both \\
\hline Metric & No & Yes & Yes \\
\hline Planner & SIADEX & $\begin{array}{l}\text { Conditional effects } \\
\text { Metrics }\end{array}$ & Memporal \\
\hline Plan compilation & Moodle/IMS-LD & Moodle/IMS-LD & Moodle/ISM-LD \\
\hline
\end{tabular}

Table 3 Comparison of the three compilations.

In summary, the Hierarchical compilation permits modelling many e-learning features, but only the planner SIADEX (Castillo et al. 2006) supports them, and it does not support quality metrics yet. The other compilations use PDDL and deal with optimisation metrics, such as maximising the learning reward. However, most state-of-the-art planners cannot maximise metrics, so a metric for maximising the total utility that the LOs report to the student cannot be always used and represents an important challenge for existing planners. From a pedagogical side, it is not easy (nor possible) to transform the metric for maximising the reward into a metric for minimising its inverse, due to how rewards are computed in this domain. In particular, we use a mapping defined in (Baldiris et al. 2008) that contains information on whether the learning resource type is good, very good or indifferent for each learning style. But we cannot assert anything about the inverse. For instance, it is untrue that if a lecture is very good for a reflective student it has to be very bad for a non-reflective student. So, it is unclear which values to assign to those cases not covered in (Baldiris et al. 2008).

The main conclusion is that there is not clearly a best approach. In some cases, the hierarchical compilation shows more natural (particularly when there are many IsPartOf relations); in others, the conditional compilation is very appealing to model and subsume all alternative branches that students may follow; and in other cases, the temporal compilation shows more expressive, because of its simulation of hierarchical structures and use of a complete numeric representation.

\section{Experimental Results}

In this section, we perform a quantitative and qualitative evaluation. From a quantitative perspective, we are interested in technical experiments to assess the correctness of the compilation schemas from a planning perspective. Thus, we have run some experiments with four courses defined in IMS-MD. The first one is a complete AI course containing LOs for covering all the typical tasks, such as reading a subject, practising, programming, performing exams, etc. Each task includes from one to six optional ones with different degrees of adequacy to the learning styles. The course has 172 LOs, 32 of them representing common tasks and 14 hierarchical aggregations. This course contains enough LOs for testing the planning complexity and for covering realistic-size courses. The other courses, Representation, Planning and Search, are subsets of this.

After compiling the IMS-MD course following the three approaches, we have defined the corresponding planning problems. All the compilation processes finished in a few seconds. We have used one planner for each compilation: SIADEX for the hierarchical domain, CBP (Fuentetaja, et al. 2009) for the Conditional domain, and LRnPlanner (Garrido \& Onaindía 2010) for the Temporal domain. We have also analysed the applicability of many state-of-the-art planners that participated in the ICAPS planning competitions (http://ipc.icaps-conference.org), but they do not support all the domain requirements at the same time, i.e. conditional effects, or and negative preconditions, fluents and cost metrics. Therefore, we have used these three planners because they support all the domain requirements for each compilation and are sound 
(all their generated plans are valid). SIADEX is a knowledge-based HTN planner with temporal features. CBP includes the original algorithms of METRIC-FF, and it also implements new heuristics and algorithms to better deal with cost metrics - the original METRIC-FF could not solve all the tested problems. LRNPLANNER deals with durative actions and metrics. We also tried to solve the durative problems with MIPS-XXL but it has scalability problems due to the numeric representation; all the information is encoded as numeric functions and current planners have problems with this kind of reasoning.

First, we performed some experiments to analyse the scalability of the approaches. We considered problems with 1, 2, 4, 8, 16, 32 and 64 students and measured the running time for solving the problems. For the longest course (AI course), the time to solve the problems ranged: in the Hierarchical approach from 0.04s for 1 student up to 97.41 s for the 64 students; in the Conditional one from $0.47 \mathrm{~s}$ to $455.94 \mathrm{~s}$; and in the Temporal one from $0.07 \mathrm{~s}$ to $37.29 \mathrm{~s}$. The execution time for the other domains were proportional to the course size and number of students (we do not show detailed results for lack of space). This means that all the approaches can cope well with a reasonable number of students. The size of the plans ranged progressively: in the Hierarchical approach from 86, $172\left(86^{*} 2\right), 258\left(86^{*} 3\right) \ldots 5504\left(86^{*} 64\right)$ actions for 1, 2, $3 . .64$ students, respectively; in the Conditional one $78,156\left(78^{*} 2\right) \ldots 4992\left(78^{*} 64\right)$ actions; and in the Temporal one from 66 to $4224\left(66^{*} 64\right)$ actions.

Second, we performed other experiments to test the quality of the plans, i.e. e-learning designs. Obviously, quality depends on the pedagogical theory used and how it is defined by the education experts. In our approach, the Hierarchical domain is based on the order in which the LOs are presented to each student, while the Conditional and Temporal domains are based on the utility (reward) theory mentioned above. There are very specific courses with evaluations based on a student satisfaction-oriented perspective (Castillo et al. 2010). But our tested courses are more generally designed and use a theory that implies the planner should find a plan that maximises the total reward without exceeding the total time the student can devote to the course. That is, the solution plan must contain as many LOs that fit the particular student profile as the time constraint allows. For example, the plan for an active student should contain LOs that the recommendation table in (Baldiris et al. 2008) suggests for active students. However, as current state-of-the-art planners cannot maximise metrics, we have transformed the reward maximisation into a penalty minimisation metric. As mentioned before, there is not an exact transformation of metric for maximising the reward into a metric for minimising its inverse, so we use an approximation. The recommendation table contains information on whether the learning resource type is good, very good or indifferent for each learning style. We use a penalty PDDL-fluent that increases in two units each time the plan includes an indifferent action, in one unit when it includes a good action, and there is not penalty for very good actions, but this can be easily modified to other values. This way, we guide the planner to include very good actions in the plan, i.e. actions that provide greater reward to the student. We vary the domain and the student profile, and measure: i) the size, i.e. number of LOs in the solution plan, ii) the total time and penalty of the learning design, and iii) the differences between plans. For these differences, we take the first plan (the one for the active student) as the base plan and count the number of LOs in the other plans that are not included in it as a measure of adaptation to students. The bigger the difference is, the more actions are found for that particular type of student that are not suggested in the baseline. In the Hierarchical approach the differences are the number of LOs that are presented to the student in a different order than the base plan. We consider problems with the four profiles in the Honey-Alonso taxonomy, i.e. active, reflexive, theoretical and pragmatic, which correspond to the active, reflective, intuitive and sensitive ones in the Felder taxonomy, respectively. Table 4 shows the results. The rows represent the planning problems: student profile (P: 1-active, 2-theoretical/intuitive, 3-pragmatic/sensitive, and 4-reflective) and courses (the first 4 rows correspond to the Representation course, the following 4 rows to the Planning course, the following rows to the Search course and the last ones to the complete AI course). Columns 
represent: number of LOs in the plan $(\mathrm{LOs})$, total time $(\mathrm{Ti})$ of the course, penalty $(\mathrm{Pe})$ and number of differences ( $\mathrm{Di}$ ). The Conditional approach uses an additional reward threshold; a plan is valid only if the total reward is equal or greater than this threshold, which poses the question of setting the initial reward threshold value. We have initially set it to zero and executed the planner. The reward obtained in the solution plan is the threshold value used in the experiments. As the base case we used the solution returned by CBP without using any metric nor reward threshold. The total time thresholds (maximum time the student can devote to the course) were bigger enough, i.e. there were not time constraints.

\begin{tabular}{|c|c|c|c|c|c|c|c|c|c|c|c|c|c|c|c|}
\hline & \multicolumn{4}{|c|}{ Base Case } & \multicolumn{4}{|c|}{ PDDL-Conditional } & \multicolumn{4}{|c|}{ PDDL-Temporal } & \multicolumn{3}{|c|}{ HTN-PDDL } \\
\hline $\mathrm{P}$ & LOs & $\mathrm{Ti}$ & $\mathrm{Pe}$ & Di & LOs & $\mathrm{Ti}$ & $\mathrm{Pe}$ & Di & LOs & $\mathrm{Ti}$ & $\mathrm{Pe}$ & $\mathrm{Di}$ & LOs & $\mathrm{Ti}$ & Di \\
\hline 1 & 15 & 650 & 10 & & 29 & 825 & 6 & & 20 & 755 & 7 & & 14 & 650 & \\
\hline 2 & 15 & 650 & 7 & 0 & 30 & 935 & 10 & 7 & 20 & 815 & 10 & 2 & 14 & 650 & 0 \\
\hline 3 & 15 & 650 & 16 & 0 & 28 & 735 & 11 & 1 & 20 & 755 & 11 & 0 & 14 & 650 & \\
\hline 4 & 15 & 650 & 7 & 0 & 30 & 845 & 10 & 6 & 20 & 755 & 11 & 0 & 14 & 650 & 0 \\
\hline 1 & 13 & 510 & 7 & & 26 & 715 & 1 & & 12 & 570 & 1 & & 13 & 600 & \\
\hline 2 & 13 & 510 & 4 & 0 & 18 & 660 & 3 & 10 & 12 & 420 & 3 & 3 & 13 & 600 & 13 \\
\hline 3 & 13 & 510 & 10 & 0 & 27 & 625 & 2 & 2 & 12 & 570 & 12 & 0 & 13 & 600 & \\
\hline 4 & 13 & 510 & 4 & 0 & 18 & 720 & 4 & 8 & 12 & 510 & 4 & 2 & 13 & 600 & 13 \\
\hline 1 & 40 & 1430 & 29 & & 49 & 1785 & 17 & & 40 & 1730 & 12 & & 44 & 1620 & \\
\hline 2 & 40 & 1430 & 14 & 0 & 51 & 1685 & 14 & 11 & 40 & 1430 & 14 & 9 & 44 & 1620 & 31 \\
\hline 3 & 40 & 1430 & 34 & 0 & 46 & 1455 & 19 & 10 & 40 & 1620 & 14 & 4 & 44 & 1620 & \\
\hline 4 & 40 & 1430 & 17 & 0 & 48 & 1595 & 15 & 10 & 40 & 1430 & 17 & 9 & 44 & 1620 & 31 \\
\hline 1 & 66 & 2530 & 45 & & 81 & 2925 & 22 & & 66 & 2655 & 26 & & 86 & 3290 & \\
\hline 2 & 66 & 2530 & 28 & 0 & 87 & 2845 & 27 & 23 & 66 & 2335 & 29 & 14 & 86 & 3290 & 55 \\
\hline 3 & 66 & 2530 & 59 & 0 & 80 & 2595 & 34 & 11 & 66 & 2705 & 37 & 2 & 86 & 3290 & \\
\hline 4 & 66 & 2530 & 32 & 0 & 84 & 2815 & 30 & 18 & 66 & 2215 & 38 & 14 & 86 & 3290 & 55 \\
\hline
\end{tabular}

Table 4 Experimental results for the adaptation of plans. Running time was less than $0.2 \mathrm{~s}$ in all cases.

The results show that the plans generated with the Conditional and Temporal approaches better adapt to the student profile. Even though there are fewer LOs in the base plans than in the others, the penalty is nearly always greater. That means that our approaches find learning designs with a higher number of LOs, i.e. the student has more possibilities to better learn the course, and most of them are very good for its profile. The Hierarchical approach also shows adaptation to the student profile according to the theory it is based on: it only distinguishes two kinds of students, pragmatic and theoretical ones (the active style is considered as pragmatic and reflective as theoretical). The plans generated for both kind of students contain several actions in different order, but the first domain is too simple to appreciate this difference.

Finally, in order to verify that the e-learning designs obtained by applying the proposed methodology are valid, i.e. correctly specified, we have compiled the plans into the e-learning standard IMS-LD and we have correctly uploaded the resulting XML files to the learning dotLRN (www.dotlrn.org) and RELOAD (http://www.reload.ac.uk) platforms.

On the other hand, a thorough qualitative evaluation of the approaches is difficult, as it involves the collaboration of educational researchers for a correct definition of the courses and for a comprehensive testing with real students. The work presented in (Castillo et al. 2010) reports some preliminary experiments in this direction for the Hierarchical compilation. The main result here is that it is not easy to measure the goodness of our approach via the students grades/scores, as following a learning design does not necessarily mean achieving a better grade. Therefore, we have designed a brief questionnaire to evaluate the quality of the learning designs, i.e. plans for the whole AI course detailed above, and their adequacy to the students profiles from the lecturer perspective. Table 5 shows the results of this evaluation by nine lecturers of AI courses, in terms of the three different compilations and two different learning styles. In particular, the first three rows (questions) refer to the votes for the plans obtained for active/pragmatic students, whereas the following three are for the reflective/theoretical ones. In general, the experts agree (though not very strongly) with the designs given by the three compilations in terms of their form, size and adaptation to the students. The main reason why there were not more strongly agree answers was that we had loosened too much the constraints (relations) among LOs, thus allowing the 
planners to generate plans with non-standard orders in the sequence of activities. Even so, the generated orders were correct with respect to the initial LOs. Also, an additional test with respect to the compilation with the most coherent and consistent sequence of LOs shows that one lecturer prefers the conditional compilation, three the temporal conditional and five the HTN one, as it seems more natural. Finally, and according to the lecturers opinion, it is extremely hard to design (and evaluate) an adequate learning design to each profile, but they agree with the usefulness of applying planning technology to help their labor.

\begin{tabular}{|c|c|c|c|c|c|c|c|c|c|c|c|c|}
\hline & Strongly disagree & & sagree & & eutr & & & $\operatorname{gre}$ & & $\mathrm{St}$ & $\mathrm{ngl}$ & agree \\
\hline Question & $\begin{array}{lll}\mathrm{C} & \mathrm{T} & \mathrm{H}\end{array}$ & $\mathrm{C}$ & $\begin{array}{ll}\mathrm{T} & \mathrm{H}\end{array}$ & $\mathrm{C}$ & $\mathrm{T}$ & $\mathrm{H}$ & $\mathrm{C}$ & $\mathrm{T}$ & $\mathrm{H}$ & $\mathrm{C}$ & $\mathrm{T}$ & $\mathrm{H}$ \\
\hline Q1 & & 2 & 1 & 2 & 3 & 4 & 4 & 4 & 4 & 1 & 1 & 1 \\
\hline Q2 & & 2 & 1 & 4 & 5 & 5 & 2 & 2 & 4 & 1 & 1 & \\
\hline Q3 & 1 & 1 & & 7 & 7 & 5 & 1 & & 4 & & 1 & \\
\hline Q1 & & 2 & 2 & 2 & 4 & 4 & 4 & 2 & 4 & 1 & 1 & 1 \\
\hline Q2 & & 1 & 3 & 6 & 4 & 5 & 1 & 2 & 4 & 1 & & \\
\hline Q3 & 1 & 1 & 1 & 7 & 6 & 3 & 1 & & 6 & & 1 & \\
\hline
\end{tabular}

Table 5 Questionnaire for a qualitative evaluation of learning designs by nine lecturers. C, $\mathrm{T}$ and $\mathrm{H}$ stand for PDDL-Conditional, PDDL-Temporal and HTN-PDDL compilations, respectively.

\section{Conclusions and Future Work}

This paper has contributed with both a general and three instantiated approaches for automatic compilations that interpret and translate e-learning models to planning. A course definition is represented as a planning domain, the student learning information as a planning problem for that domain and the learning design as the plan generated by a domain-independent planner. Our three different compilations of planning domains and problems allow current planners to automatically generate valid learning designs. Our experimental results have raised challenges and shown scalability limitations in some cases. Also, a complete analysis of our compilations has allowed us to detect three important drawbacks that show semantic gaps between the pedagogical decisions and the automated translations. First, instructional designs are complex to model; they tend to be theory-independent and do not capture the pedagogical knowledge required to generate a course. Second, e-learning languages are usually too generic and try to cover too many aspects, making the implementation of general and suitable translators for all LMSs very difficult and, in some cases, slightly imprecise (e.g. the learning resource types). Third, despite the expressive power of e-learning languages, there are still some aspects that cannot be represented and are essential in P\&S. For example, the definition of the resources involved in the tasks, their costs, the temporal constraints on availability and how these resources are to be managed are important lacks in e-learning languages. In other words, e-learning standards still miss some information for pedagogically interesting planning. Analogously, a more flexible approach for requiring/achieving the learning outcomes is also missing. For instance, the execution of a task may result in a higher effect in one student than in another, depending on the student learning style. Informally, two students do not learn the same with the same task, and the definition of this type of conditional effects should be available in the e-learning standards. We have solved this problem by implementing pedagogical suggestions into the compilers. But all in all, our compilations show very appropriate for integrating e-learning standards and AI planning, while also providing the strong foundations to be extended to other particular education theories.

In the future, we intend to overcome the previous drawbacks by addressing three parallel lines. Firstly, coming up with more expressive models of actions for planning e-learning activities, thus augmenting the semantics of the model, which will increase the opportunities to: i) deal with complex course composition, and ii) validate and resolve courses with similar but incommensurate LOs. Secondly, extending our system to assist the course designer in making sure that the 
same naming conventions are used by adopting a standard or common ontology, as proposed in (Kontopoulos et al. 2008), thus avoiding unsolvable situations (e.g. having a LO requiring knowledge on planners and a student knowing about planning systems). Thirdly, working on the actual execution of courses and the dynamic planning adaptation of courses with respect to the real behavior of students.

\section{Acknowledgements}

This work has been supported by the Spanish MICINN under projects TIN2008-06701-C03 and Consolider Ingenio 2010 CSD2007-00022, by the Mexican National Council of Science and Technology and the regional projects CCG08-UC3M/TIC-4141 and Prometeo GVA 2008/051.

\section{References}

Alonso, C. \& Honey, P. (2002). 'Honey-Alonso Learning Style Theoretical Basis (in Spanish)'. Available at http://www.estilosdeaprendizaje.es/menuprinc2.htm (acces. Nov. 2010).

Baldiris, S. et al. (2008). 'Integration of Educational Specifications and Standards to Support Adaptive Learning Scenarios in ADAPTAPlan'. Special Issue on New Trends on AI techniques for Educational Technologies. International Journal of Computer Science and Applications (IJCSA) 5(1):88-107.

Boticario, J. \& Santos, O. (2007). 'A Dynamic Assistance Approach to Support the Development and Modelling of Adaptive Learning Scenarion Based on Educational Standards'. In Proc. of Workshop on Authoring of Adaptive and Adaptable Hypermedia, Int. Conference on User Modelling, pp. 1-8.

Camacho, D. et al. (2007). 'CAMOU: a Simple Integrated e-Learning and Planning Techniques Tool'. In 4 th International Workshop on Constraints and Language Processing, pp. 1-11.

Castillo, L. et al. (2006). 'Efficiently handling temporal knowledge in an HTN planner'. In Proc. of 16th Int. Conference on Automated Planning and Scheduling (ICAPS 2006), pp. 63-72. AAAI.

Castillo, L. et al. (2010). 'Automatic generation of temporal planning domains for e-learning problems'. Journal of Scheduling 13(4):347-362.

Essalmi, F. et al. (2010). 'A fully personalization strategy of E-learning scenarios'. Computers in Human Behavior 26(4):581-591.

Felder, R. M. (1996). 'Matters of Style'. ASEE Prism 6(4):18-23.

Fox, M. \& Long, D. (2003). 'PDDL2.1: an Extension to PDDL for Expressing Temporal Planning Domains'. Journal of Artificial Intelligence Research 20:61-124.

Fuentetaja, R. et al. (2009). 'A Look-ahead B\&B Search for Cost-Based Planning'. In Proc. of CAEPIA'09, pp. 105-114.

Garrido, A. \& Onaindía, E. (2010). 'On the Application of Planning and Scheduling Techniques to Elearning'. In Proc. 23rd Int. Conference on Industrial, Engineering 6 Other Applications of Applied Intelligent Systems (IEA-AIE 2010) - LNCS, pp. 244-253. Springer.

Garrido, A. et al. (2009). 'Modeling E-Learning Activities in Automated Planning'. In Proc. of the 3rd Int. Competition on Knowledge Engineering for Planning and Scheduling (ICKEPS-2009), pp. 18-27.

IMSLD (2003). 'IMS Learning Design Specification. Version 1.0 (February, 2003)'. Available at http://www.imsglobal.org/learningdesign (acces. Nov. 2010).

IMSMD (2003). 'IMS Learning Resource Meta-data Specification. Version 1.3 (August, 2006)'. Available at http://www.imsglobal.org/metadata (acces. Nov. 2010).

Kontopoulos, E. et al. (2008). 'An Ontology-based Planning System for E-course Generation'. Expert Systems with Applications 35:398-406.

Limongelli, C. et al. (2008). 'LS-Plan: an Effective Combination of Dynamic Courseware Generation and Learning Styles in Web-Based Education'. In Adaptive Hypermedia and Adaptive Web-Based Systems, 5th International Conference, AH 2008, pp. 133-142. Springer Berlin / Heidelberg.

Méndez, N. et al. (2005). 'IA planning for automatic generation of customized virtual courses'. Frontiers In Artificial Intelligence And Applications. Planning, scheduling and constraint satisfaction: from theory to practice 117:139-148.

Mohan, P. et al. (2003). 'Instructional Planning with Learning Objects'. In IJCAI-03 Workshop Knowledge Representation and Automated Reasoning for E-Learning Systems, pp. 52-58.

SCORM (2004). 'Sharable Content Object Reference Model'. Available at http://www.adlnet.gov/Technologies/scorm/default.aspx (acces. Nov. 2010).

Sicilia, M. et al. (2006). 'On Supporting the Process of Learning Design through Planners'. CEUR Workshop Proceedings: Virtual Campus 2006 Post-Proceedings. 186(1):81-89.

Ullrich, C. (2008). Pedagogically Founded Courseware Generation for Web-Based Learning. No. 5260. LNAI, Springer.

Ullrich, C. \& Melis, E. (2009). 'Pedagogically Founded Courseware Generation based on HTN-Planning'. Expert Systems With Applications 36(5):9319-9332. 